\title{
Adiponectin complexes in human cerebrospinal fluid: distinct complex distribution from serum
}

\author{
C. M. Kusminski • P. G. McTernan • T. Schraw • \\ K. Kos • J. P. O'Hare • R. Ahima • S. Kumar • \\ P. E. Scherer
}

Received: 2 September 2006 / Accepted: 21 November 2006 / Published online: 23 January 2007

(C) Springer-Verlag 2007

\begin{abstract}
Aims/hypothesis Adiponectin is an adipocyte-derived secretory factor that is specifically produced in adipocytes. It exerts effects on energy homeostasis via peripheral and central mechanisms. However, it is not clear whether adiponectin crosses the blood-brain barrier in humans. In serum, adiponectin circulates in several different complexes, each of which has distinct functions. Here, we wanted to test whether adiponectin can be found in human
\end{abstract}

C. M. Kusminski • P. G. McTernan • K. Kos • J. P. O’Hare •

S. Kumar

Unit for Diabetes and Metabolism, Warwick Medical School,

University of Warwick, Clinical Sciences Research Institute,

UHCW Campus,

Coventry, UK

C. M. Kusminski • T. Schraw $\cdot$ P. E. Scherer $(\triangle)$

Department of Cell Biology, Chanin Rm 515,

Albert Einstein College of Medicine,

1300 Morris Park Avenue,

Bronx, NY 10461, USA

e-mail: scherer@aecom.yu.edu

R. Ahima

Department of Medicine, Division of Endocrinology, Diabetes and Metabolism, University of Pennsylvania School of Medicine, Philadelphia, PA, USA

R. Ahima

Institute for Diabetes, Obesity and Metabolism,

Philadelphia, PA, USA

P. E. Scherer

Department of Medicine, Albert Einstein College of Medicine, Bronx, NY, USA

\section{P. E. Scherer}

Diabetes Research and Training Center,

Albert Einstein College of Medicine,

Bronx, NY, USA cerebrospinal fluid (CSF) and whether specific adiponectin complexes are enriched in CSF compared with peripheral serum samples. We also wanted to establish whether there is a sex-related difference with regard to the distribution of adiponectin oligomers in CSF.

Materials and methods We studied 22 subjects (11 men, 11 women) in this study. Their average BMI was $28.0 \pm$ $4.7 \mathrm{~kg} / \mathrm{m}^{2}$; average age was $70 \pm 7$ years.

Results Analysis of total adiponectin revealed that adiponectin protein is present in human CSF at approximately $0.1 \%$ of serum concentration. The distribution of adiponectin oligomers differs considerably in CSF from that of serum within matched samples from the same patients. Only the adiponectin trimeric and low-molecular-mass hexameric complexes are found in CSF, with a bias towards the trimeric form in most patients. Male subjects have a higher CSF: serum ratio of total adiponectin $(p<0.05 ; n=20)$ and have slightly higher trimer levels in serum and CSF than female subjects.

Conclusions/interpretation We conclude that the adiponectin trimer is the predominant oligomer in human CSF.

Keywords Adipokines - Adiponectin · Blood-brain barrier . Human $\cdot$ Metabolic syndrome $\cdot$ Obesity/energy homeostasis

$\begin{array}{ll}\text { Abbreviations } \\ \text { ADIPOR } & \text { adiponectin receptor } \\ \text { BBB } & \text { blood-brain barrier } \\ \text { CNS } & \text { central nervous system } \\ \text { CSF } & \text { cerebrospinal fluid } \\ \text { HMW } & \text { high molecular weight } \\ \text { HOMA- } & \text { homeostasis model assessment of insulin } \\ \text { IR } & \text { resistance } \\ \text { i.c.v. } & \text { intracerebroventricular } \\ \text { LMW } & \text { low molecular weight }\end{array}$


$\mathrm{S}_{\mathrm{A}} \quad$ adiponectin sensitivity index

TBS-T Tris-buffered saline containing $0.1 \%$ Tween 20

\section{Introduction}

Adipose tissue secretes numerous proteins that can exert multiple effects through the central nervous system (CNS) and peripheral tissues to modulate energy homeostasis [1]. Leptin is one of these adipokines to have been extensively studied in this context. It serves as a potent satiety signal to the brain and regulates adiposity by suppressing appetite, increasing insulin sensitivity and influencing other anorexigenic hormones that mediate energy expenditure [1-3]. Translocation of leptin across the blood-brain barrier (BBB) is modulated through a saturable transport system, consistent with a role of $\mathrm{BBB}$ in regulating behaviour and maintaining CNS homeostasis [1, 4].

Adiponectin is an adipocyte-specific secretory protein involved in numerous aspects of energy homeostasis [5]. Many studies have reported a strong correlation between increased circulating levels of adiponectin and improved insulin sensitivity [6-9]. Furthermore, circulating adiponectin levels are reduced in states of obesity-induced insulin resistance, while weight reduction triggers an increase in adiponectin levels [7, 10]. Systemic adiponectin treatment or transgenic adiponectin overexpression in rodents improves metabolic status, enhancing the ability of insulin to suppress hepatic glucose production [6-9, 11]. In contrast, adiponectin-null mice exhibit hepatic insulin resistance [12]. Collectively, this suggests that the primary in vivo process targeted by adiponectin action in the context of insulin sensitisation is hepatic glucose homeostasis. Consistent with these in vivo observations, primary hepatocytes exposed to recombinant adiponectin respond by reducing cellular glucose output as a function of adiponectin even at very low insulin concentrations. However, several recent papers have highlighted the need for both peripheral and central insulin action on hepatic glucose output $[13,14]$. Similarly, adiponectin may exert its action both peripherally and centrally.

Adiponectin consists of an N-terminal collagenous domain and a C-terminal globular domain $[6,7]$ that shares homology with types VIII and X collagen, in addition to complement factor $\mathrm{C} 1 \mathrm{q}[6,7]$. When secreted, adiponectin circulates in serum in distinct stable forms, as trimers and low-molecular-weight (LMW) hexamers, or as high-molecular-weight (HMW) multimeric complexes, comprising 12-18 subunits $[6,15]$. A critical cysteine residue in position 39 is the key mediator of oligomerisation beyond the basic trimeric building block [6].
Previous studies have documented a characteristic sexual dimorphism in adiponectin levels in humans, with female subjects having significantly higher circulating levels than male subjects [16]. This sexual dimorphism is also reflected in differential levels of the various circulating adiponectin complexes. The hexamer is the more prevalent form of adiponectin in male subjects, whereas female subjects have higher circulating levels of the HMW complex; since the HMW form has been implicated as the key complex in the context of insulin sensitisation, this may be the source of increased insulin sensitivity of female subjects compared with male subjects [6].

The adiponectin Cys-39 mutant with its destabilised homotrimeric structure shows greater bioactivity in vivo than the native oligomeric complexes in terms of its potency to reduce serum glucose levels [6]; in addition, the recombinant globular, collagenous stalk-free form of adiponectin influences lipid oxidation in peripheral skeletal muscle tissues [11], with limited effects on hepatic gluconeogenesis [17]. However, the globular form of the protein has not yet been identified under normal physiological conditions in vivo. The native full-length adiponectin complex exerts more potent effects on the liver. Previous studies have demonstrated that transgenic mice overexpressing endogenous full-length adiponectin display substantial improvement in hepatic insulin sensitivity [18]. It is also recognised that the ratio of HMW to LMW oligomeric forms of adiponectin measured by the adiponectin sensitivity index $\left(\mathrm{S}_{\mathrm{A}}\right)$, rather than total circulating levels of adiponectin, is a critical determinant of thiazolidinedionemediated improvements in hepatic insulin sensitivity [19], diabetic patients having decreased $\mathrm{S}_{\mathrm{A}}$ values, which improve with thiazolidinedione treatment [19]. The HMW oligomers are thus established as important adiponectin forms with regard to hepatic insulin sensitivity.

A direct functional impact of adiponectin on metabolism, effected by its enhancing of insulin sensitivity, is therefore well established. However, recent studies have shed light on another central role of adiponectin, demonstrating that adiponectin has profound effects on energy homeostasis in the brain. Intracerebroventricular (i.c.v.) administration of globular, full-length or Cys-39Ser mutant adiponectin into wild-type and $o b / o b$ mice decreased body weight, the effect being mediated via increased energy expenditure [20]. Furthermore, peripherally administered adiponectin caused an increase in cerebrospinal fluid (CSF) adiponectin, suggesting serum-to-CSF transport [20]. However, these studies measured the adiponectin levels by RIA in mice only and lacked a more detailed examination of the specific complex distribution of adiponectin. Central treatment with adiponectin proved more potent than systemic treatment of adiponectin in wild-type and $o b / o b$ mice, further highlighting the brain as an important target for adiponectin action 
[20]. In addition, adiponectin was shown to induce Fos protein immunostaining and increase hypothalamic corticotrophin-releasing hormone synthesis in the paraventricular nucleus, suggesting the activation of hypothalamic sympathetic circuits [20]. Further evidence suggesting a role for adiponectin in the CNS originates from several studies reporting the levels of adiponectin receptors 1 and 2 (ADIPOR1 and ADIPOR2) in the brain [21] and, more specifically, in mouse hypothalamus and on brain endothelial cells of the BBB [22]; the latter may potentially serve as a transport system for receptor-mediated transcytosis of adiponectin across the BBB. However, a more specific role for these receptors remains to be defined.

These studies suggest that adiponectin may act centrally to exert metabolic actions. However, the question of whether adiponectin plays a role centrally under normal physiological conditions has yet to be resolved. One recent study failed to detect the presence of adiponectin in human CSF [22] and, with another, reported that exogenous adiponectin does not cross the BBB in mice [22, 23], concluding that direct effects of adiponectin on CNS pathways may not be relevant.

The focus of our study was to firmly establish the presence of adiponectin in human CSF and subsequently to establish the distribution of the various oligomers by means of sub-fractionation and quantitative western blot analysis of CSF samples.

\section{Subjects and methods}

\section{Subjects}

We analysed 22 subjects, all of whom were white, aged $58-81$ years and with a BMI of $28.0 \pm 4.7 \mathrm{~kg} / \mathrm{m}^{2}$. Half of the subjects were male (BMI $28.1 \pm 3.8 \mathrm{~kg} / \mathrm{m}^{2}$; age range $58-$ 80 years) and half were female (all post-menopausal; BMI $28.0 \pm 5.7 \mathrm{~kg} / \mathrm{m}^{2}$; age range $62-81$ years). CSF and matched serum samples were obtained from these consenting subjects, who were undergoing elective surgery. All samples were acquired in accordance with the Ethics Committee of the Birmingham Heartlands Hospital (Birmingham, UK) and analysed at the Albert Einstein College of Medicine (Bronx, NY, USA) under a protocol approved by the Institutional Review Board. Patients with malignancy, acute and chronic renal or liver disease, or neurological disorders, as well as those on immunosuppressants, current or recent systemic high-dose corticosteroids, antibiotics or weight altering medication were excluded from the study. All subjects were fasted overnight before examination. The sampling of CSF was performed according to standardised procedures with the examined subject in a lateral recumbent position and lumbar puncture at the L3-L4 or L5-L6 interspace using a standard needle. Prior to spinal anaesthesia injection, a clear volume of CSF was extracted. CSF samples were passed through a 0.2 micron syringe filter (Whatman, Florham Park, NJ, USA), divided into aliquots, flash frozen and stored at $-80^{\circ} \mathrm{C}$ until analysis. A fasting blood sample $(5 \mathrm{ml})$ was also taken at the time of venous cannulation. Samples were immediately centrifuged at 5,000 $\mathrm{g}$ for $20 \mathrm{~min}$, flash frozen and stored at $-80^{\circ} \mathrm{C}$.

Size fractionation of adiponectin complexes using fast protein liquid chromatography

Analysis of serum samples Once thawed, $30 \mu \mathrm{l}$ of each serum sample was spun at $10,000 \mathrm{~g}$ for $10 \mathrm{~min}$. Next $20 \mu \mathrm{l}$ of column buffer ( $25 \mathrm{mmol} / \mathrm{l}$ HEPES, $150 \mathrm{mmol} / \mathrm{l} \mathrm{NaCl}$, $1 \mathrm{mmol} / 1 \mathrm{CaCl}_{2} ; \mathrm{pH} 8$ ) was added to each serum sample. The sample mixture was then injected into a GL column (Superdex 200 10/300; GE Healthcare Bio-Sciences, Piscataway, NJ, USA).

Analysis of CSF samples We added $20 \mu \mathrm{l}$ of adiponectin knockout mouse serum to $200 \mu \mathrm{l}$ of CSF sample as a carrier. The mixture was then spun at $10,000 \mathrm{~g}$ for $10 \mathrm{~min}$ before being injected undiluted on to the column. In total, $200-\mu \mathrm{l}$ gradient-fractions were sequentially retrieved for each CSF and serum sample. For CSF fractions, $200 \mu \mathrm{l}$ of each fraction was vacuum concentrated for $1 \mathrm{~h}$ (SpeedVac Plus; Savant Instruments, Holbrook, NY, USA). Fractions were analysed by quantitative western blot analysis as described below.

\section{Immunoblotting}

In brief, for serum fractions, $20 \mu \mathrm{l}$ of $5 \times$ Laemmli sample buffer was added to $60 \mu \mathrm{l}$ of each fraction then heated at $95^{\circ} \mathrm{C}$ for $20 \mathrm{~min}$. Lyophilised CSF fractions were reconstituted in $25 \mu \mathrm{l}$ sample buffer then heated at $95^{\circ} \mathrm{C}$ for 5 min. Separation of proteins and immunoblotting was performed as previously described [6]. Following SDSPAGE, proteins were transferred on to a polyvinylidene fluoride membrane (Millipore, Billerica, MA, USA), then blocked with $4 \%$ non-fat dried milk in Tris-buffered saline containing $0.1 \%$ Tween 20 (TBS-T) for $1 \mathrm{~h}$. Primary antibodies specifically raised against the human N-terminal hypervariable region of adiponectin (DQETTTQGPGV) and the human $\mathrm{C}$-terminal region of adiponectin was diluted $(1: 200)$ in TBS-T; these antibodies recognise a single band of $30 \mathrm{kDa}$ by western blot analysis and have equal affinity for all oligomeric forms of adiponectin. Membranes were washed four times for $5 \mathrm{~min}$ in $0.1 \%$ TBS-T. CSF blots were additionally probed with a biotinconjugated goat anti-rabbit antibody (1:500; Invitrogen, 
Carlsbad, CA, USA). To allow for visualisation of bands, CSF blots were decorated with a fluorescent streptavidinconjugate secondary antibody (1:5,000; Invitrogen), while serum blots were decorated with a fluorescent dyeconjugated anti-rabbit secondary antibody (Rockland Immunochemicals, Gilbertsville, PA, USA), diluted $1: 5,000$, in $0.1 \%$ TBS-T. Fractions 1-8 (HMW adiponectin), 9-16 (LMW adiponectin) and 16-25 (adiponectin trimer) from sedimentation were visualised at $700 \mathrm{~nm}$ for CSF blots and $800 \mathrm{~nm}$ for serum blots using a Phosphor Imager (LI-COR Biosciences, Lincoln, NE, USA), then quantified using Odyssey Licor System software (LI-COR Biosciences, Lincoln, NE, USA). Total serum and CSF adiponectin levels were measured by RIA (Linco Research, St Charles, MO, USA; lower detection limit of $1 \mathrm{ng} / \mathrm{ml}$ for $100-\mu l$ sample size). Total insulin levels were determined by ELISA (Linco Research). Glucose levels were determined using a glucose analyser (YSI-2300 STAT PLUS; Yellow Springs Instruments, Yellow Springs, OH, USA), in accordance with the manufacturers' instructions. Calculation of homeostasis model assessment of insulin resistance (HOMA-IR) [24] was performed using a HOMA-IR calculator obtained from http://www.dtu.ox.ac.uk/index. php? maindoc $=/$ homa/index.php.

\section{Statistics}

The SPSS statistical program, version 14.0 for Windows (SPSS, Woking, UK) was used to analyse data. Results are expressed as percentage or mean \pm standard deviation. A two-tailed Student's $t$ test was used to assess differences between serum and CSF adiponectin oligomers in male and female patients as these were Gaussian in distribution. A bivariate Pearson's correlation coefficient was used to analyse associations between the following variables: (1) $\log$ serum adiponectin; (2) $\log$ CSF adiponectin; (3) BMI and (4) HOMA-IR. A $p$ value of $<0.05$ was considered statistically significant.

\section{Results}

Total serum and CSF levels of adiponectin by western blot analysis

To date, adiponectin levels in CSF have only been reported as RIA measurements. Using western blot analysis, we demonstrate that a single $30-\mathrm{kDa}$ band cross-reactive with highly specific anti-adiponectin antibodies can be found in human CSF. In parallel, we analysed a corresponding serum sample from the same patient (Fig. 1). Note that the intensities should not be directly compared between the two western blots. The relative ratio of CSF:serum adiponectin is given for each patient based on the western blot quantitation. This ratio varies from patient to patient, suggesting that the levels in CSF are actively controlled. We subsequently measured the serum and CSF levels by radioimmunoassay. In absolute terms, the abundance of adiponectin is much lower in CSF, namely approximately $0.1 \%$ of the levels found in serum (serum $=14.0 \pm 5.7$ $[\mathrm{mean} \pm \mathrm{SD}] \mu \mathrm{g} / \mathrm{ml} ; \mathrm{CSF}=11.9 \pm 18.8 \mathrm{ng} / \mathrm{ml}[n=22])$. RIA analysis further revealed that generally, higher levels of adiponectin in serum tend to be associated with higher levels of adiponectin in CSF; this correlation was significant in men $(p=0.044 ; r=0.615 ; n=11)$ but not in women ( $p=\mathrm{NS} ; r=0.263 n=11$ ) (Fig. 2).
Fig. 1 Total adiponectin in serum and matched CSF by western blot analysis. The CSF: serum adiponectin ratio is given for each patient analysed $(n=20)$. Representative western blots are shown for total serum adiponectin and corresponding total CSF adiponectin for each patient. Patient sex: $M$, male patient; $F$, female patient
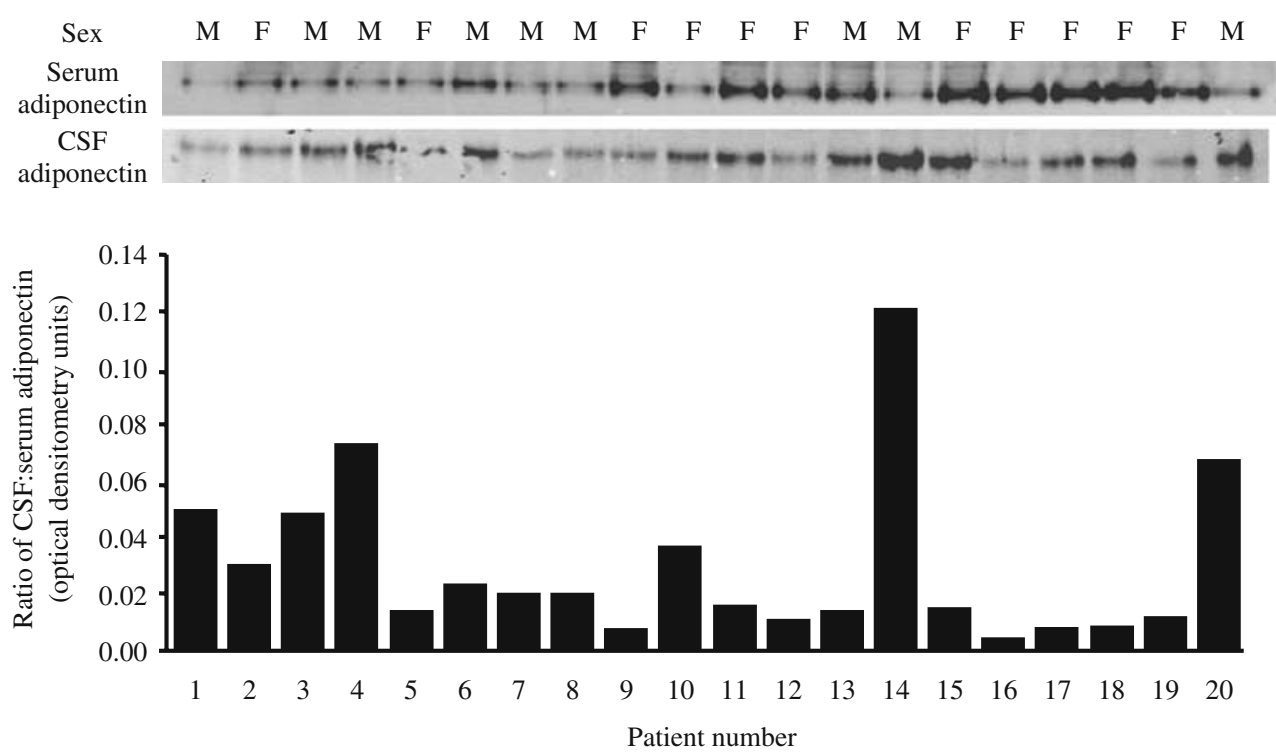


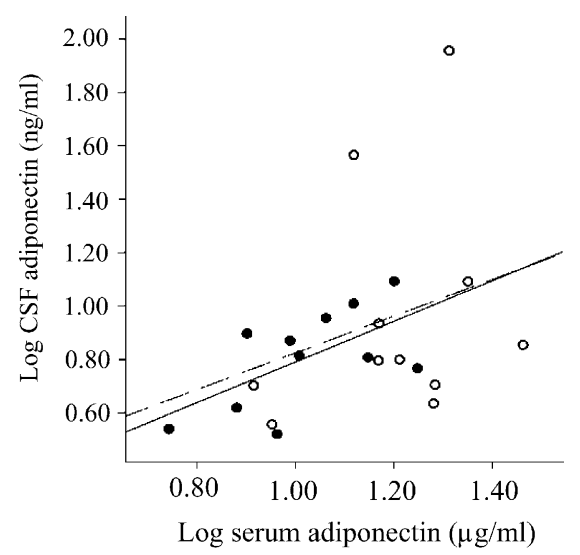

Fig. 2 Total and CSF adiponectin levels in male patients (solid symbols $)(p=0.044 ; r=0.615 ; n=11)$ and female patients (open symbols) ( $p=\mathrm{NS} ; r=0.263 ; n=11)$ were measured by RIA. Solid line, trend line for male patients; dashed line, trend line for female patients

Trimer and LMW hexamer forms of adiponectin are the predominant complexes found in human CSF

Following sub-fractionation of CSF by gel filtration chromatography and subsequent quantitative western blotting, the distribution of adiponectin oligomers in human CSF was visualised (Fig. 3); only the adiponectin LMW hexamer, and more prevalently the trimer were found in CSF. A comparison of serum and CSF adiponectin oligomer distribution revealed that adiponectin displays a diverse oligomeric complex distribution of oligomers in CSF compared with the matched serum within the same patient. Serum showed the expected distribution of HMW complexes, LMW hexamers and the adiponectin trimers. CSF, in contrast, lacked the HMW form prominent in serum.
While a single representative example is shown in Fig. 3, we expanded the analysis to an additional 13 samples. For serum analysis, we calculated the fraction of each adiponectin oligomer relative to the total adiponectin levels. The average percentage composition of adiponectin oligomers in serum across a range of patients in the study was: HMW $40.2 \pm 12.5 \%$; LMW $35.6 \pm 6.8 \%$; and trimer $24.2 \pm 8.9 \%$ $(n=13)$ (Table 1). The analysis of CSF revealed: no detectable HMW; LMW 19.2 $\pm 9.1 \%$; and trimer $80.8 \pm$ $9.1 \%$. The lack of the HMW form of adiponectin enabled us to exclude the possibility that CSF could have been contaminated by serum.

Sexual dimorphism of total adiponectin and oligomeric complex distribution

Further analysis of total adiponectin levels in serum compared with CSF, using western blot, revealed that male subjects have a significantly higher CSF:serum ratio of adiponectin than female subjects $(p<0.05 ; n=20)$ (Fig. 4). As previously established, women have higher levels of HMW adiponectin in serum than men. In contrast, men have slightly higher levels of trimeric adiponectin in serum than women. Although Table 1 demonstrates such a trend towards sex-related differences in serum HMW and trimeric adiponectin ( $p=\mathrm{NS}$; men, $n=6$; women, $n=7$ ), further analysis using a larger cohort may substantiate significance. No significant differences were detected at the level of LMW adiponectin hexamer $(p=\mathrm{NS})$ (Table 1). Although men displayed slightly higher levels of adiponectin trimer in CSF than women, this did not reach statistical significance ( $p=\mathrm{NS}$; men, $n=6$; women, $n=7$ ) (Table 1). Additional studies will therefore be required to see whether these trends can be confirmed in larger cohorts.
Fig. 3 The distribution of adiponectin oligomers in CSF (solid line) in comparison with that in matched serum (dotted line) from one female patient $(n=13)$. The average percentage composition of each adiponectin oligomer in serum is: HMW $40.2 \%$; LMW 35.6\%; trimer $24.2 \%(n=13)$. The average percentage composition of adiponectin trimer and LMW hexamer in CSF is: LMW $19.2 \%$; trimer $80.8 \%$. Representative western blots from the same female patient following size fractionation highlight serum and CSF adiponectin oligomer distribution
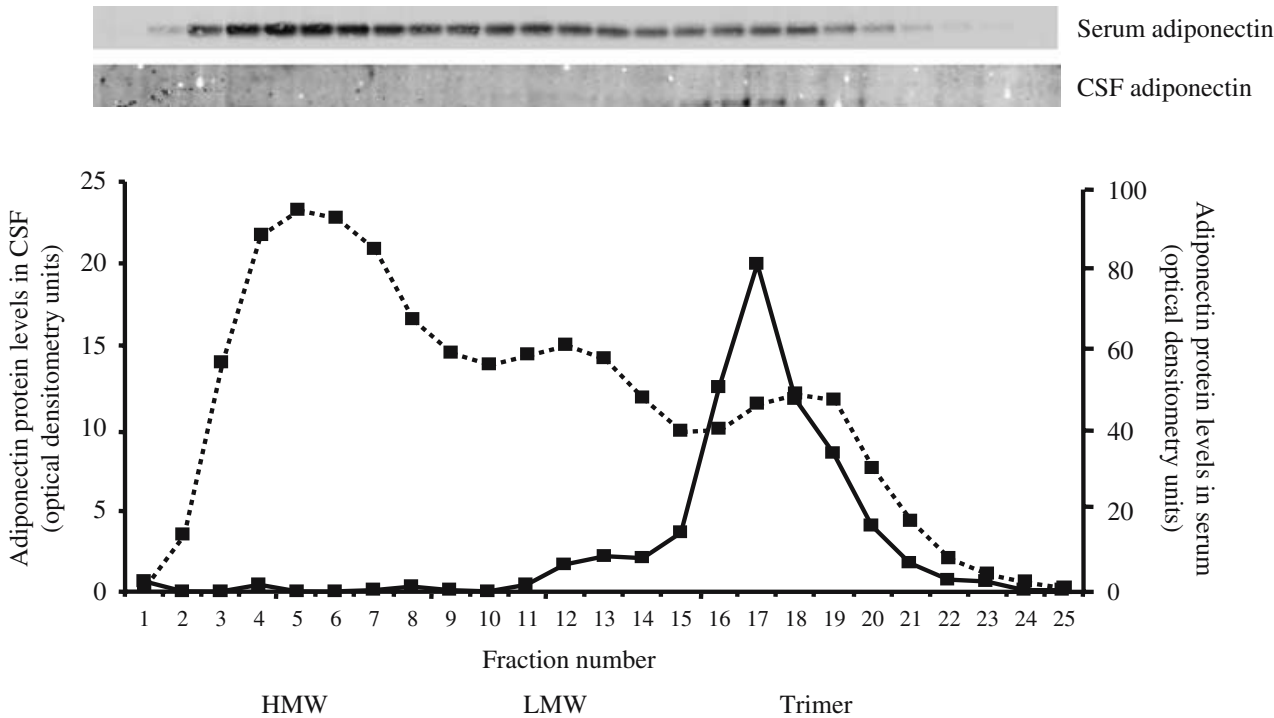
Table 1 Average percentage compositions of each adiponectin oligomer in serum and CSF in male patients $(n=6)$, female patients $(n=7)$ and in both combined

\begin{tabular}{|c|c|c|c|c|c|c|}
\hline \multirow[b]{2}{*}{ Adiponectin oligomer } & \multicolumn{2}{|c|}{ Male patients $(n=6)( \pm \mathrm{SD})$} & \multicolumn{2}{|c|}{ Female patients $(n=7)( \pm \mathrm{SD})$} & \multicolumn{2}{|c|}{ Total patients $(n=13)( \pm \mathrm{SD})$} \\
\hline & Serum & $\mathrm{CSF}$ & Serum & $\mathrm{CSF}$ & Serum & $\mathrm{CSF}$ \\
\hline HMW & $36.5 \pm 11.3$ & Not measurable & $43.4 \pm 13.4$ & Not measurable & $40.2 \pm 12.5$ & Not measurable \\
\hline LMW & $35.4 \pm 9.1$ & $17.6 \pm 5.2$ & $35.9 \pm 4.7$ & $20.6 \pm 11.8$ & $35.6 \pm 6.8$ & $19.2 \pm 9.1$ \\
\hline Trimer & $28.2 \pm 5.4$ & $82.4 \pm 5.2$ & $20.8 \pm 10.2$ & $79.4 \pm 11.8$ & $24.2 \pm 8.9$ & $80.8 \pm 9.1$ \\
\hline
\end{tabular}

No statistical differences in HMW, LMW or trimeric adiponectin were reached between male patients and female patients. $H M W$ high molecular weight, $L M W$ low molecular weight

\section{Correlation with BMI and HOMA-IR}

As in many previous studies, we found that levels of total adiponectin in serum are inversely related to BMI. In our study, RIA analysis revealed that total serum adiponectin significantly correlates with BMI in male patients ( $p=0.047$; $r=-0.609 ; n=11$ ) (Fig. 5a); however, this was not the case in the female patients ( $p=\mathrm{NS} ; r=-0.329 ; n=11)$ (Fig. 5a). Furthermore, when examining the correlation between CSF adiponectin and BMI in men and women, analysis revealed a trend, albeit not significant, towards an inverse relationship of CSF adiponectin with BMI (male patients, $p=0.067$; $r=-0.571 ; n=11$; female patients, $p=\mathrm{NS} ; r=-0.116 ; n=11$ ) (Fig. 5b).

Within our cohort, glucose and insulin concentrations were $6.0 \pm 1.7 \mathrm{mmol} / 1$ and $9.4 \pm 13.3 \mu \mathrm{U} / \mathrm{ml}(n=22)$ respectively. When examining insulin resistance index, serum adiponectin levels were shown to inversely correlate with the HOMA-IR index in male patients only (male patients, $p=0.014 ; r=-0.744 ; n=10$; female patients, $p=\mathrm{NS}$; $r=-0.504 ; n=11)$. Similarly, CSF adiponectin demonstrated a significant correlation with HOMA-IR in male patients, although not in female patients (male patients, $p=0.037$; $r=-0.663 ; n=10$; female patients, $p=\mathrm{NS} ; r=-0.330 ; n=11$ ).

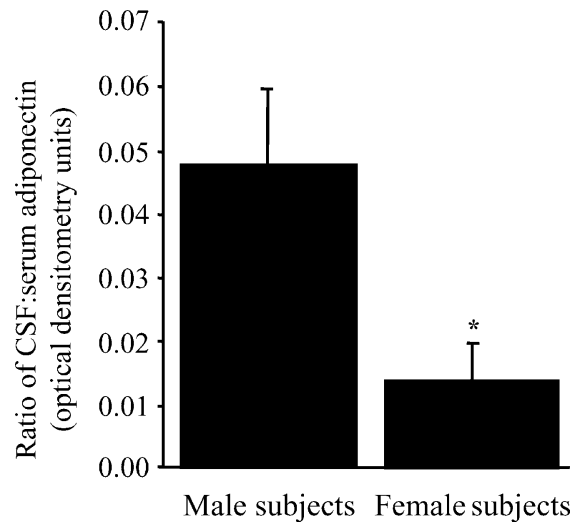

Fig. 4 The ratio of total CSF:serum adiponectin in male patients compared with female patients by western blot analysis. ${ }^{*} p<0.05$; $n=20$

\section{Discussion}

Here we establish the presence of adiponectin in human CSF, confirming the ability of adiponectin to traverse the BBB and further identifying its potential to function centrally under normal physiological conditions. A key finding in our study was the diverse oligomeric distribution between CSF and serum within matched samples from the same patients. While all forms of adiponectin were present in serum, only the trimer and LMW hexamer were present in CSF; in particular, the adiponectin trimer was substantially more prevalent in CSF than the LMW form. Interestingly, male subjects displayed a higher CSF:serum ratio of total adiponectin than female subjects. Subfractionation analysis of serum further revealed that men had slightly more adiponectin trimer than women; although not significant, men also had slightly higher levels of adiponectin trimer in CSF than women. As in many previous studies, the LMW hexamer does not seem to be regulated in any significant fashion.

Our study thus shows that the trimer is the primary form of adiponectin in human CSF. This further supports previous observations that i.c.v. injection of wild-type, Cys39Ser mutant or globular adiponectin had a central effect, while the collagenous tail domain lacking the globular head domain was ineffective [20]. Intracerebroventricular administration of adiponectin proved to have a more potent effect on energy homeostasis than systemic administration of equivalent amounts of adiponectin [20].

A number of recent papers highlight the relevance of the HMW form for the peripheral functions of adiponectin [19, 25-30]. In light of the very large size of this complex $(>500 \mathrm{kDa})$, it is not surprising that the HMW form is very inefficiently translocated across the BBB and not found in any measurable quantities in CSF. The HMW form, despite its relevance for peripheral adiponectin action, does not seem to play a major physiological role in central adiponectin action. In contrast, the trimeric form of adiponectin seems to be most relevant for the central action of this protein. 

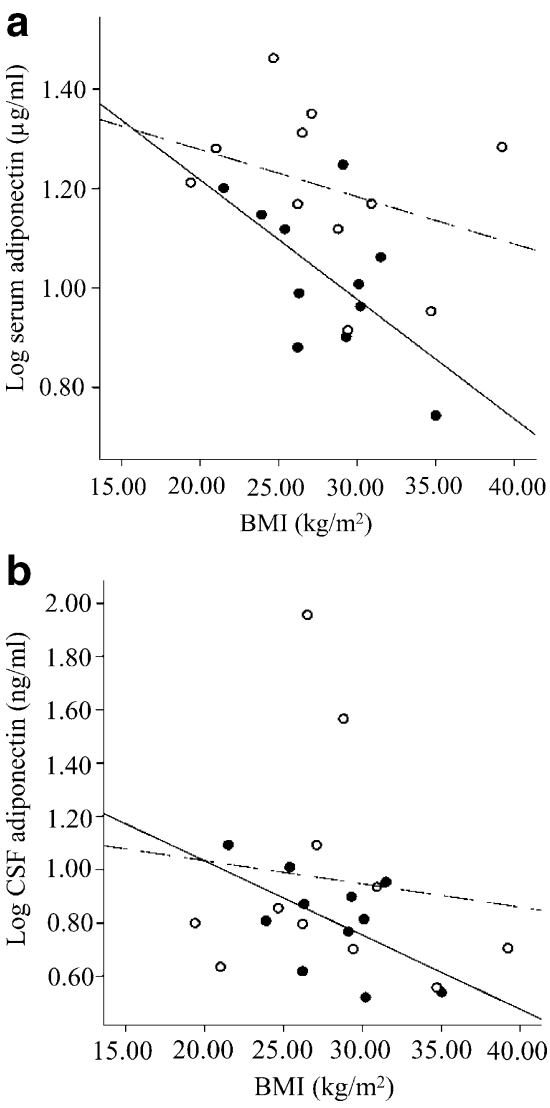

Fig. 5 a Correlation of total serum adiponectin with BMI in male patients (solid symbols) $(p=0.047 ; r=-0.609 ; n=11)$ and female patients (open symbols) ( $p=\mathrm{NS} ; r=-0.329 ; n=11)$ by RIA. b Correlation of total CSF adiponectin with BMI in male patients $(p=0.067 ; r=-0.571 ; n=11)$ and female patients ( $p=\mathrm{NS} ; r=-0.116 ; n=11)$ by RIA

The BBB plays a major dynamic regulatory role in the passage of circulating peptides involved in energy homeostasis [4]. How, exactly, the adiponectin trimer crosses the BBB is currently unknown. Adipor1 and Adipor2 have been detected in the brain [21] and on BBB endothelial cells [22], suggesting that a regulated receptor-mediated transport system may be the favourable mechanism of entry. However, the identification and kinetic properties of adiponectin transporters remains to be determined.

Leptin concentrations in CSF are positively related to plasma level and BMI, but the CSF:plasma leptin ratio is reduced at the highest plasma leptin level, suggesting a saturable brain transport mechanism [31, 32]. The decrease in CSF:plasma leptin among obese patients is consistent with leptin resistance $[31,32]$. CSF leptin levels in children account for $5 \%$ of plasma levels, but unlike the plasma level, which is higher in girls, the CSF leptin concentration is similar in girls and boys [33]. Thus, the CSF:plasma leptin ratio is lower in girls, and this may underlie the propensity towards excess body fat [33]. A nocturnal plasma leptin surge entrained to meal timing has been demonstrated in lean and obese humans [34, 35]. However, this is not accompanied by an increase in CSF leptin [36].
As leptin transport across the BBB is fully saturated at higher serum leptin concentrations, it is possible that the BBB serves as a rate-limiting step to prevent increases in CSF leptin concentrations. The BBB could thus contribute to 'leptin resistance'; such a notion has been reported in obese subjects, where the increases in plasma leptin were not followed by parallel increases in the CSF leptin levels $[31,32]$. How the lack of a nocturnal increase in CSF leptin relates to the periodicity of energy balance and neuroendocrine axis is unknown [36].

Similar mechanisms may apply for adiponectin. Indeed, Kim and colleagues recently reported that a mouse model expressing a dominant negative version of the IGF-1 receptor transgenically in muscle displayed complete adiponectin resistance [37]. Future experiments will have to address more specifically the mechanism of adiponectin BBB transport under different physiological conditions.

Adipor 1, preferential for globular adiponectin and trimeric adiponectin, is abundantly expressed in skeletal muscle, mediating AMP-activated protein kinase activation to increase glucose uptake and lipid oxidation [21]. Adipor2, on the other hand, which favours full-length adiponectin, is more highly expressed in the liver, mediating hepatic glucose homeostasis [21]. Interestingly, higher levels of ADIPOR1 than ADIPOR2 are found in the brain [21], consistent with our findings that the adiponectin trimer is the predominant CSF form. Whether BBB endothelial cells specifically express higher levels of Adipor1 is, however, uncertain.

The relatively low overall protein concentrations in CSF and the low abundance of adiponectin in CSF make the biochemical analysis challenging. In addition, the concentrations found in CSF may or may not be sufficiently high to bind and activate the known ADIPORs, given the affinity constants reported for the receptors. However, it is difficult to rule out the possibility that other mechanisms are in place to increase the local concentrations of the adiponectin trimer to levels that would effectively trigger receptor activation. Alternatively, additional, yet-to-be-identified receptors may exist that display higher affinities.

We used antibodies that specifically recognise human adiponectin and do not show any cross-reactivity with murine adiponectin. To avoid introducing an exogenous source of adiponectin altogether, we used serum from adiponectin null mice as a carrier during the biochemical subfractionation of the CSF samples, a step that proved to be essential for the efficient detection of CSF adiponectin.

Putative transport systems for both leptin and TNF- $\alpha$ have been identified or postulated to exist [4]; these transport systems should be able to independently facilitate receptor-mediated transcytosis of peptides across the $\mathrm{BBB}$ [38]. Whether such a scenario exists for adiponectin transport is currently unknown. Once across the BBB, the 
influence of adiponectin on peripheral energy expenditure may be mediated by the hypothalamus. Adiponectin induces c-fos expression in the paraventricular nucleus [20] and increases the synthesis of hypothalamic corticotrophin-releasing hormone [20], suggesting activation of adiponectin-responsive neurons via the melanocortin pathway. Agouti $A^{\mathrm{y}} / a$ mice, incapable of melanocortin signalling [39], are resistant to central administration of adiponectin [40], further indicating that the melanocortin pathway is essential for the anorexigenic effects of adiponectin on energy expenditure. Leptin $o b / o b$ mice are particularly sensitive to CNS and systemic adiponectin treatment [9, 20], suggesting perhaps a compensatory role for adiponectin to counteract reduced leptin signalling in the melanocortin pathway. Furthermore, mice lacking leptin receptors only in proopiomelanocortin neurons have a modest increase in body weight [41]. Future studies examining mice lacking ADI PORs in pro-opiomelanocortin neurons may prove illuminating and may establish whether leptin and adiponectin act in concordance to activate such neurons.

Western blot analysis of total adiponectin revealed that male subjects had a higher CSF:serum ratio than female subjects. Sexual dimorphism in terms of total systemic adiponectin, with women having higher levels of adiponectin than men, has been reported [10, 42, 43]. Although this was preserved in CSF [18], such findings were achieved using assay-based measures. As it is currently unknown whether available RIAs and ELISAs are biased towards some adiponectin oligomers, total adiponectin data should be interpreted with care. Our study further confirms the sexual dimorphism of adiponectin in terms of the oligomeric complex distribution in serum and, to a lesser extent, in CSF. Men have slightly higher levels of the trimeric form of adiponectin in circulation than women; this is partly reflected in CSF as well.

The three oligomeric adiponectin complexes have differential secretion rates [44], possibly dependent on the status of energy expenditure and on peripheral and hepatic insulin sensitivity. The rate of HMW adiponectin secretion from adipocytes is slower than that of the other two oligomeric complexes [44]. Testosterone has been shown to selectively impede secretion of the HMW form of adiponectin from adipocytes, possibly by activating intracellular sequestering molecules [44]. Interestingly, testosterone elevates the relative levels of trimeric and hexameric adiponectin forms at the expense of the HMW form in circulation [44], consistent with the increased susceptibility of men to hepatic insulin resistance. Androgens may therefore serve as one of many factors modulating the pattern of adiponectin oligomeric complex distribution within target tissues. Consequently, healthy women displaying higher levels of HMW adiponectin also display improved hepatic insulin sensitivity compared with men. In addition, post-menopausal women may exhibit a more androgenic setting of adiponectin oligomers in comparison with young pre-menopausal women. Future examination of menopausal status in terms of adiponectin oligomer distribution in serum and CSF may further define the role of hormonal factors as modulators of adiponectin.

A recent paper has reported negative data regarding the presence and/or transport of adiponectin into CSF [22]. It is unclear why the authors were unable to detect adiponectin in CSF. However, given the negative data reported for the transport of bacterially produced globular trimer in these studies, it is possible that the collagenous stalk or some additional post-translational modification associated with it may be important for the transport process.

Several key questions remain outstanding with regard to central adiponectin action. What factors determine the distribution of adiponectin forms between CSF and the periphery? Is this distribution altered in obese or insulinresistant patients, i.e. is there a specific defect in the BBB translocation machinery under some pathological conditions? Does the nutritional status affect this distribution? Future studies examining adiponectin oligomeric distribution in CSF in diabetic or in chronically obese patients, as well as pre- and post-peroxisome proliferator-activated receptor- $\gamma$ agonist intervention treatment, may clarify the mechanisms underlying central adiponectin action.

In conclusion, adiponectin is present in human CSF, predominantly in the trimeric form. More specifically, CSF adiponectin employs a distinct oligomeric pattern to that of serum and is further modulated by sex.

Acknowledgements C. M. Kusminski was supported by a European Foundation for the Study of Diabetes Travel Fellowship and a Diabetes UK PhD Studentship. We would like to thank members of the Scherer laboratory for their excellent technical advice and support, in particular Y. Cho for help at various stages of the project. P. E. Scherer was supported by National Institutes of Health grant R03 EY014935-01 and is also a recipient of an Irma T. Hirschl Career Scientist Award.

Duality of interest None of the authors report any conflicts of interest related to the data presented here.

\section{References}

1. Ahima RS, Flier JS (2000) Adipose tissue as an endocrine organ. Trends Endocrinol Metab 11:327-332

2. Friedman JM, Halaas JL (1998) Leptin and the regulation of body weight in mammals. Nature 395:763-770

3. Elmquist JK, Elias CF, Saper CB (1999) From lesions to leptin: hypothalamic control of food intake and body weight. Neuron $22: 221-232$

4. Zlokovic BV, Jovanovic S, Miao W et al (2000) Differential regulation of leptin transport by the choroid plexus and bloodbrain barrier and high affinity transport systems for entry into hypothalamus and across the blood-cerebrospinal fluid barrier. Endocrinology 141:1434-1441 
5. Scherer PE, Williams S, Fogliano M et al (1995) A novel serum protein similar to C1q, produced exclusively in adipocytes. J Biol Chem 270:26746-26749

6. Pajvani UB, Du X, Combs TP et al (2003) Structure-function studies of the adipocyte-secreted hormone Acrp30/adiponectin. Implications for metabolic regulation and bioactivity. J Biol Chem 278:9073-9085

7. Tsao TS, Lodish HF, Fruebis J (2002) ACRP30, a new hormone controlling fat and glucose metabolism. Eur $\mathrm{J}$ Pharmacol 440:213-221

8. Yamauchi T, Kamon J, Waki H et al (2001) The fat-derived hormone adiponectin reverses insulin resistance associated with both lipoatrophy and obesity. Nat Med 7:941-946

9. Berg AH, Combs TP, Du X et al (2001) The adipocyte-secreted protein Acrp30 enhances hepatic insulin action. Nat Med 7:947953

10. Combs TP, Berg AH, Rajala MW et al (2003) Sexual differentiation, pregnancy, calorie restriction, and aging affect the adipocyte-specific secretory protein adiponectin. Diabetes 52:268-276

11. Yamauchi T, Kamon J, Waki H et al (2003) Globular adiponectin protected ob/ob mice from diabetes and ApoE-deficient mice from atherosclerosis. J Biol Chem 278:2461-2468

12. Nawrocki AR, Rajala MW, Tomas E et al (2006) Mice lacking adiponectin show decreased hepatic insulin sensitivity and reduced responsiveness to peroxisome proliferator-activated receptor gamma agonists. J Biol Chem 281:2654-2660

13. Okamoto H, Obici S, Accili D et al (2005) Restoration of liver insulin signaling in Insr knockout mice fails to normalize hepatic insulin action. J Clin Invest 115:1314-1322

14. Cherrington $\mathrm{AD}$ (2005) The role of hepatic insulin receptors in the regulation of glucose production. J Clin Invest 115:1136-1139

15. Berg AH, Combs TP, Scherer PE (2002) ACRP30/adiponectin: an adipokine regulating glucose and lipid metabolism. Trends Endocrinol Metab 13:84-89

16. Arita Y, Kihara S, Ouchi N et al (1999) Paradoxical decrease of an adipose-specific protein, adiponectin, in obesity. Biochem Biophys Res Commun 257:79-83

17. Fruebis J, Tsao TS, Javorschi S et al (2001) Proteolytic cleavage product of $30-\mathrm{kDa}$ adipocyte complement-related protein increases fatty acid oxidation in muscle and causes weight loss in mice. Proc Natl Acad Sci USA 98:2005-2010

18. Combs TP, Pajvani UB, Berg AH et al (2004) A transgenic mouse with a deletion in the collagenous domain of adiponectin displays elevated circulating adiponectin and improved insulin sensitivity. Endocrinology 145:367-383

19. Pajvani UB, Hawkins M, Combs TP et al (2004) Complex distribution, not absolute amount of adiponectin, correlates with thiazolidinedione-mediated improvement in insulin sensitivity. J Biol Chem 279:12152-12162

20. Qi Y, Takahashi N, Hileman SM et al (2004) Adiponectin acts in the brain to decrease body weight. Nat Med 10:524-529

21. Yamauchi T, Kamon J, Ito Y et al (2003) Cloning of adiponectin receptors that mediate antidiabetic metabolic effects. Nature 423:762-769

22. Spranger J, Verma S, Gohring I et al (2006) Adiponectin does not cross the blood-brain barrier but modifies cytokine expression of brain endothelial cells. Diabetes 55:141-147

23. Pan W, Tu H, Kastin AJ (2006) Differential BBB interactions of three ingestive peptides: obestatin, ghrelin, and adiponectin. Peptides 27:911-916

24. Mathews DR, Hosker JP, Rudenski AS et al (1985) Homeostasis model assessment: insulin resistance and beta-cell function from fasting plasma glucose and insulin concentrations in man. Diabetologia 28:412-419

25. Waki H, Yamauchi T, Kamon J et al (2003) Impaired multimerization of human adiponectin mutants associated with diabetes.
Molecular structure and multimer formation of adiponectin. J Biol Chem 278:40352-40363

26. Tonelli J, Li W, Kishore P et al (2004) Mechanisms of early insulin-sensitizing effects of thiazolidinediones in type 2 diabetes. Diabetes 53:1621-1629

27. Fisher FF, Trujillo ME, Hanif W et al (2005) Serum high molecular weight complex of adiponectin correlates better with glucose tolerance than total serum adiponectin in Indo-Asian males. Diabetologia 48:1084-1087

28. Wang Y, Lam KS, Xu JY et al (2005) Adiponectin inhibits cell proliferation by interacting with several growth factors in an oligomerization-dependent manner. J Biol Chem 280:18341-18347

29. Lara-Castro C, Luo N, Wallace P et al (2006) Adiponectin multimeric complexes and the metabolic syndrome trait cluster. Diabetes 55:249-259

30. Hara K, Horikoshi M, Yamauchi T et al (2006) Measurement of the high-molecular weight form of adiponectin in plasma is useful for the prediction of insulin resistance and metabolic syndrome. Diabetes Care 29:1357-1362

31. Schwartz MW, Peskind E, Raskind M et al (1996) Cerebrospinal fluid leptin levels: relationship to plasma levels and to adiposity in humans. Nat Med 2:589-593

32. Caro JF, Kolaczynski JW, Nyce MR et al (1996) Decreased cerebrospinal-fluid/serum leptin ratio in obesity: a possible mechanism for leptin resistance. Lancet 348:159-161

33. Wiedenhoft A, Muller C, Stenger R et al (1999) Lack of sex difference in cerebrospinal fluid (CSF) leptin levels and contribution of $\mathrm{CSF} /$ plasma ratios to variations in body mass index in children. J Clin Endocrinol Metab 84:3021-3024, Erratum in: J Clin Endocrinol Metab (1999) 84:4171

34. Sinha MK, Ohannesian JP, Heiman ML et al (1996) Nocturnal rise of leptin in lean, obese, and non-insulin-dependent diabetes mellitus subjects. J Clin Invest 97:1344-1347

35. Schoeller DA, Cella LK, Sinha MK et al (1997) Entrainment of the diurnal rhythm of plasma leptin to meal timing. J Clin Invest 100:1882-1887

36. Wong ML, Licinio J, Yildiz BO et al (2004) Simultaneous and continuous 24-hour plasma and cerebrospinal fluid leptin measurements: dissociation of concentrations in central and peripheral compartments. J Clin Endocrinol Metab 89:258-265

37. Kim CH, Pennisi P, Zhao H et al (2006) MKR mice are resistant to the metabolic actions of both insulin and adiponectin: discordance between insulin resistance and adiponectin responsiveness. Am J Physiol Endocrinol Metab 291:E298-E305

38. Pan W, Akerstrom V, Zhang J et al (2004) Modulation of feedingrelated peptide/protein signals by the blood-brain barrier. J Neurochem 90:455-461

39. Dinulescu DM, Cone RD (2000) Agouti and agouti-related protein: analogies and contrasts. J Biol Chem 275:6695-6698

40. Masaki T, Chiba S, Yasuda T et al (2003) Peripheral, but not central, administration of adiponectin reduces visceral adiposity and upregulates the expression of uncoupling protein in agouti yellow (Ay/a) obese mice. Diabetes 52:2266-2273

41. Balthasar N, Coppari R, McMinn J et al (2004) Leptin receptor signaling in POMC neurons is required for normal body weight homeostasis. Neuron 42:983-991

42. Nishizawa H, Shimomura I, Kishida K et al (2002) Androgens decrease plasma adiponectin, an insulin-sensitizing adipocytederived protein. Diabetes 51:2734-2741

43. Cnop M, Havel PJ, Utzschneider KM et al (2003) Relationship of adiponectin to body fat distribution, insulin sensitivity and plasma lipoproteins: evidence for independent roles of age and sex. Diabetologia 46:459-469

44. Xu A, Chan KW, RL Hoo et al (2005) Testosterone selectively reduces the high molecular weight form of adiponectin by inhibiting its secretion from adipocytes. J Biol Chem 280:18073-18080 